\title{
Dual-Antenna Dual-Band High Performance Cubesat-Compatible GPS Receiver
}

\author{
S. Rodríguez, J. García, G. Scillone, J. Díaz, E. López, and R. López
}

\begin{abstract}
Nanosatellites are revolutionizing the space industry allowing low-cost educational, scientific or commercial space missions. Among them, the CubeSats play a key role due to their standardization and increasingly use. As the CubeSat missions become more and more complex and challenging, so do their subsystems. In particular, the position, velocity and attitude of the satellite are commonly required to perform orbital tracking, precise pointing towards some sector of the Earth and other relevant tasks. These requirements can be fulfilled using an appropriate GPS receiver.

This work presents the design and development of a Global Positioning System (GPS) receiver (including hardware, software, and programmable logic) oriented to CubeSats along with some experimental results obtained with the first integrated prototype. The receiver can process the L1 and L2 GPS civil signals providing, in addition to the Position Velocity and Time (PVT) solution, high-precision pseudorange and carrier-phase measurements. It also has two antenna inputs that allow obtaining a partial attitude estimation or using the receiver as a GPS-based remote sensing sensor. The experimental results obtained from absolute and differential positioning, and partial attitude estimation show the correct working of the receiver and the excellent quality of the generated measurements.
\end{abstract}

Index Terms-GPS receiver, FPGA, multi-band, multiconstellation, CubeSat.

\section{INTRODUCCIÓN}

$\mathbf{E}$ L desarrollo de misiones espaciales de bajo costo se encuentra fuertemente impulsado por los nanosatélites. A diferencia de los satélites más tradicionales, que resultan ser voluminosos y mucho más costosos debido al uso de componentes de grado espacial requeridos para misiones de larga duración, los nanosatélites ofrecen una alternativa económicamente viable para diversas aplicaciones de investigación y de índole comercial [1]-[4].

El diseño constructivo de los nanosatélites pondera el bajo volumen, tiempo de desarrollo y el uso de componentes comerciales (COTS). Esto implica que el tiempo de misión, o tiempo de vida promedio del nanosatélite, sea de algunos meses y no de años, como en los satélites tradicionales [1].

El formato más popular de nanosatélites es el de los CubeSats, desarrollado en la Universidad Politécnica de California hace alrededor de veinte años [5]. Este formato, basado en una o más unidades cúbicas de $10 \mathrm{~cm}$ de lado es adecuado para colocar en órbita varios nanosatélites a partir de un único

This work was supported by the following institutions: Universidad Nacional de La Plata (UNLP), Consejo Nacional de Investigaciones Científicas y Técnicas (CONICET) y Agencia Nacional de Promoción Científica y Tecnológica (ANPCyT).

Authors are with SENyT, Departamento de Electrotecnia, Facultad de Ingeniería, Universidad Nacional de La Plata, La Plata, 1900, Argentina, email: santiago.rodriguez@ing.unlp.edu.ar. lanzamiento, disminuyendo el costo más importante de una misión de este tipo [6]. Según la complejidad del satélite y de la misión a desarrollar pueden combinarse unidades para formar satélites de mayor volumen, siendo comunes las configuraciones de tres o seis unidades. La forma cúbica favorece el almacenaje de módulos apilables encargados de procesar datos, administrar potencia o de realizar las demás tareas de la misión en particular [1][5].

Existen diversas aplicaciones donde puede ser útil o necesario que un nanosatélite conozca con precisión su ubicación. Un ejemplo puede ser el seguimiento orbital de la trayectoria del satélite. Otra aplicación de interés puede estar relacionada con la toma de fotos a partir de una cámara a bordo del vehículo, donde adquiere sentido conocer la ubicación para saber, con una dada precisión, qué región del planeta alcanza la cámara. Otro ejemplo es el de las arquitecturas satelitales segmentadas, donde es necesario conocer la posición y velocidad, tanto absoluta como relativa, de los miembros de la constelación con el fin de que la red satelital pueda funcionar correctamente. Una aplicación de arquitectura segmentada se presenta en la toma de imágenes con radar de apertura sintética (SAR) distribuido, donde es necesario conocer la posición de los satélites para obtener una correcta integración de la imagen conjunta [7].

Para que un satélite pueda desempeñarse en las aplicaciones mencionadas, el vehículo debe contar con un receptor de sistemas de navegación por satélite (GNSS) [2]. Los receptores GNSS reciben señales en una o más bandas de frecuencia provenientes de constelaciones de satélites de órbita media (MEO) con el fin de determinar la posición y velocidad de un usuario o vehículo. El hecho de que un receptor pueda recibir y procesar señales de distintas bandas de frecuencias permite que la solución de posicionamiento obtenida cuente con mayor precisión y robustez que en el caso mono-frecuencia [8]. De los GNSS actuales, el Sistema de Posicionamiento Global (GPS) es el más conocido y confiable debido a que se encuentra completamente operativo desde 1994.

Existen receptores GNSS comerciales orientados exclusivamente al segmento de los pequeños satélites, algunos con factor de forma compatible con el estándar CubeSat [9]-[12]. Estos receptores son extremadamente costosos, normalmente operan en una única frecuencia y poseen sólo una entrada de antena. En consecuencia, no proveen buena exactitud en la solución de posición, lo cual puede ser una limitación importante dependiendo del tipo de misión.

En este trabajo se presenta el diseño de un receptor de desarrollo propio que cuenta con dos antenas independientes y es capaz de recibir y procesar en cada una de ellas señales GPS 
de las bandas L1 y L2. Disponer de dos antenas permite incorporar funcionalidades adicionales como el cálculo parcial de orientación y lo vuelve apto para aplicaciones científicas como la reflectometría (GNSS-R) o la radio-ocultación (GNSS-RO). Otra característica distintiva del receptor propuesto es que, a diferencia de la mayoría de los receptores comerciales de bajo costo, genera mediciones crudas (pseudorangos y fase de portadora) de alta calidad que son fundamentales para posicionamiento de alta precisión.

El resto del trabajo se organiza de la siguiente manera. En la Sección II se detalla el diseño del receptor. En la sección III se muestran los ensayos realizados junto con los resultados obtenidos. Finalmente, en la Sección IV se presentan las conclusiones.

\section{DisEÑO DEL RECEPTOR}

El diseño del receptor puede separarse en tres áreas principales: el diseño del hardware, el diseño de la etapa de procesamiento digital y el diseño del software. La etapa de procesamiento digital se realiza en un hardware dedicado utilizando como plataforma un dispositivo lógico programable del tipo FPGA (Field Programmable Gate Array). Por su parte, el software se ejecuta sobre un microprocesador embebido e implementado en la misma FPGA.

\section{A. Diseño del Hardware}

El diseño propuesto, cuya arquitectura es ampliamente utilizada en receptores GNSS, se basa en dos bloques principales: una etapa de radiofrecuencia (RF) y una plataforma de procesamiento digital [13]. La etapa de RF se encarga de amplificar y filtrar adecuadamente las señales provenientes de las antenas que posteriormente ingresan a un circuito integrado que realiza la conversión a frecuencia intermedia (FI) y el muestreo correspondiente de hasta cuatro canales independientes. La etapa de procesamiento digital recibe las muestras, realiza las correlaciones necesarias para adquirir los satélites presentes en la antena, demodula los datos y calcula la solución de navegación (posición y velocidad), que se envía a otro dispositivo mediante una interfaz de comunicación.

En la Fig. 1 puede observarse un diagrama en bloques del diseño funcional del receptor. La señal de cada antena es amplificada y separada en dos canales por un divisor de potencia. Cada canal es filtrado con un filtro SAW sintonizado para las bandas L1 y L2 de GPS respectivamente. Las señales filtradas son convertidas a FI y muestreadas a dos bits, representación que utiliza un bit para el signo y otro bit para magnitud. Dichas muestras ingresan a un SoC (System on Chip) sintetizado en una FPGA.

El SoC se encuentra formado por lógica sintetizada adhoc para el cálculo de las correlaciones necesarias y por un procesador en el que se ejecuta un sistema operativo de tiempo real que comanda las acciones de todo el receptor. Para la implementación del SoC, la placa cuenta con memorias tipo SRAM y FLASH. Para fines de depuración en laboratorio se cuenta con interfaces JTAG y USB-UART. En cuanto a las interfaces de comunicación el diseño cuenta con una interfaz RS-422, una UART con niveles lógicos CMOS, una interfaz

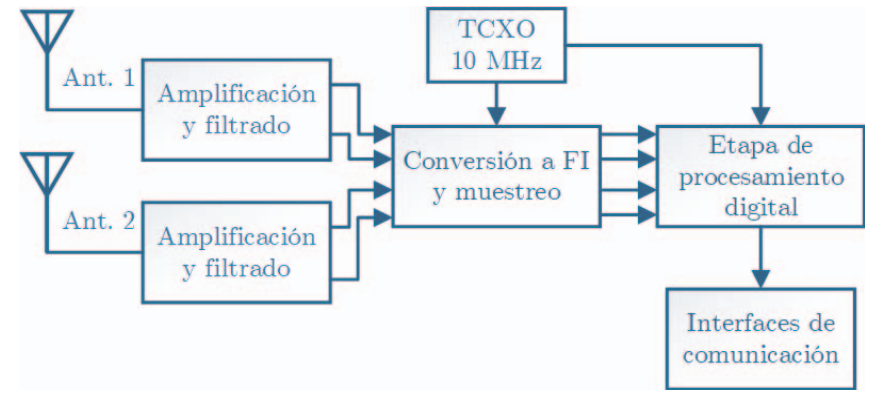

Fig. 1. Diagrama en bloques del diseño de hardware propuesto.

I2C y dos interfaces CAN, todas usualmente utilizadas en Cubesats y nanosatélites en general.

Para lograr compatibilidad mecánica con módulos comerciales para CubeSats el receptor sigue el factor de forma indicado en [14]. Para la compatibilidad eléctrica se utilizaron dos conectores de 52 pines siguiendo el estándar PC-104. Este último es normalmente utilizado para la interconexión de los módulos de un CubeSat formando una estructura vertical al colocarse uno encima de otro. El número de módulos determina el volumen que deberá tener el satélite, el cual se mide en unidades de CubeSat (denominados U) siendo $1 \mathrm{U}$ $10 \times 10 \times 10 \mathrm{~cm}$. Algunas configuraciones usuales de Cubesats son las de 1, 2, 3 y $6 \mathrm{U}$.

El diseño del PCB (Printed Circuit Board) consta de diez capas en un sustrato de material FR4. En detalle se utilizaron cuatro capas de señal, siendo dos de ellas internas, cuatro planos de tierra para minimizar acoplamiento inductivo y dos planos de alimentación, utilizados para distribuir las tensiones necesarias para el receptor priorizando la integridad de señal [15]. Los planos de tierra se encuentran intercalados entre capas de señal y por encima y por debajo de los planos de alimentación, formando un esquema que favorece la integridad de las señales. La mayor parte de los componentes se colocaron sobre la capa superior, dejando la capa inferior mayormente para componentes pasivos, como capacitores y resistencias.

El ancho de pista promedio utilizado es de 6 mils, debido principalmente al encapsulado de la FPGA, del tipo BGA de 484 bolas. Todas las vías utilizadas son de tipo pasante. Las mismas poseen un diámetro de agujero de $0.2 \mathrm{~mm}$ y una corona de $0.46 \mathrm{~mm}$ de diámetro. Para el caso de las líneas de entrada de antena, se fijó una impedancia característica de 50 $\Omega$. Para ello, se implementó una línea de transmisión de tipo microtira en la capa superior utilizando un ancho de pista de 7 mils. Otras señales de importancia en cuanto a su integridad de señal son las de reloj de sistema y muestreo, así como los pares diferenciales de USB y RS-422. En estas líneas se realizaron simulaciones para asegurar la integridad de las señales.

Para verificar que el diseño no poseía interferencias mecánicas de ningún tipo se integró el modelo 3D del receptor junto a modelos 3D de la estructura típica de un CubeSat. De esta manera, también se verificó la correcta vinculación mecánica del receptor con otros módulos disponibles en el mercado que utilicen el mismo estándar de diseño. En una primera etapa, se envió a fabricar el diseño del PCB y se ensambló parcialmente un primer prototipo con el fin de probar 


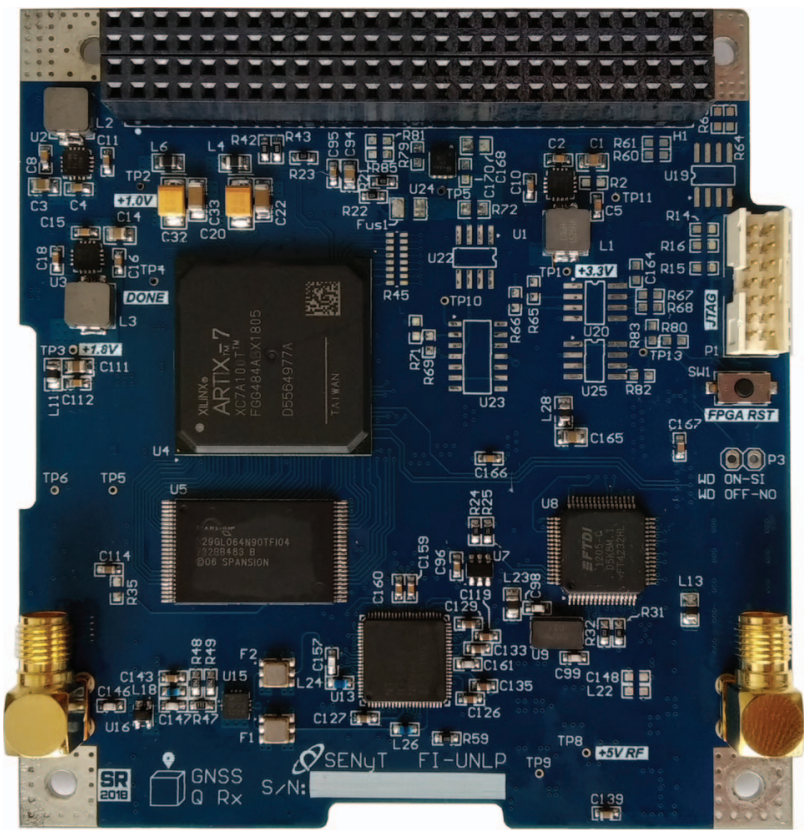

Fig. 2. Foto del prototipo construido.

y validar la etapa de recepción y procesamiento de las señales GPS de interés. En la Fig. 2 se muestra una foto del prototipo ya integrado.

\section{B. Diseño Lógico de la FPGA}

La Fig. 3 muestra el diagrama en bloques del diseño lógico del receptor junto con las interfaces de comunicación externas. La arquitectura está basada en el procesador LEON3, que ejecuta el software del receptor y controla todos los periféricos e interfaces externas. El bus AMBA es el encargado de gestionar la comunicación entre los periféricos y el procesador. Entre los principales periféricos se incluyen un controlador de memoria, que permite el acceso a las memorias RAM y FLASH, una interfaz para depuración (DSU), un controlador de interrupciones (IRQ CTRL), un temporizador de tiempo real (RTC) y varios controladores para las diferentes interfaces de comunicación: UART, CAN, SPI e I2C.

Tanto el LEON3 como los periféricos hasta aquí mencionados forman parte de las librerías de código abierto GRLIB ampliamente difundidas y muy utilizadas en este tipo de aplicaciones [16]. Adicionalmente, se incluyen periféricos desarrollados por el grupo de trabajo que cumplen tareas específicas y le otorgan funcionalidades adicionales al receptor. Entre ellos se pueden mencionar el controlador de watchdog por hardware, que permite reinicializar el receptor ante una eventual falla del software, y un módulo de adquisición de muestras en frecuencia intermedia que puede utilizarse para obtener muestras crudas sin procesar directamente de las salidas de los ADCs del cabezal de RF con fines de depuración y monitoreo [17]. Por último, el Módulo de Correlación es el encargado de procesar las muestras de GPS provenientes del cabezal de RF y volcar los resultados de las correlaciones en el bus AMBA para ser accedidos por el software del receptor.

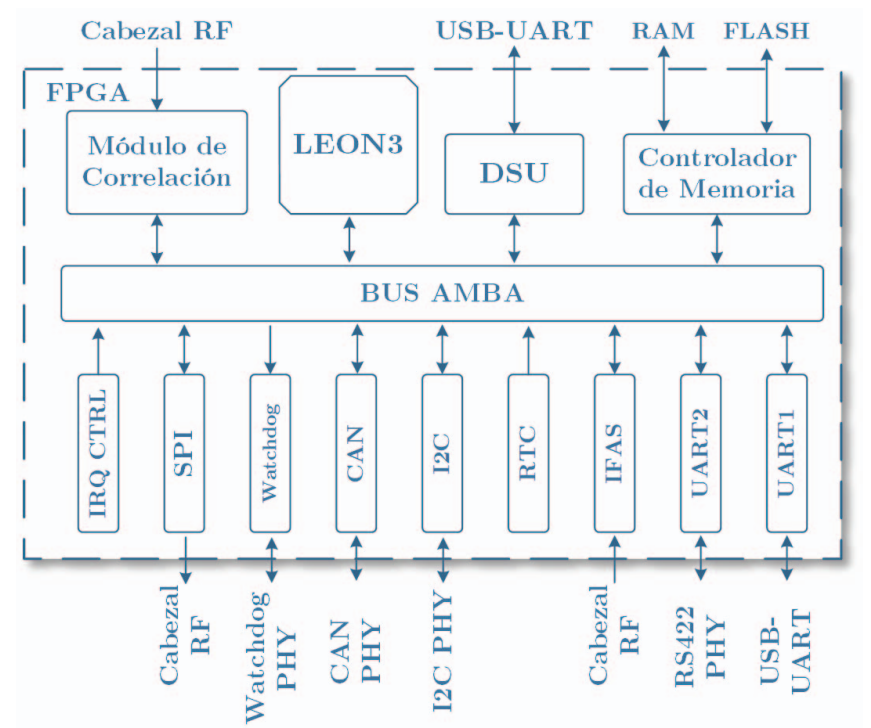

Fig. 3. Diseño lógico de alto nivel de la FPGA

Toda comunicación entre el procesador LEON3 y los periféricos, o aún entre periféricos mismos, se realiza bajo el mecanismo de mapeo en memoria. Cada periférico posee un rango de memoria asignado donde se mapean los parámetros de configuración y los datos accesibles para otros periféricos. Cuando se accede a una dirección de memoria el controlador del bus AMBA identifica a qué periférico corresponde dicha dirección y activa las señales de habilitación correspondientes a ese periférico para realizar la transacción.

El diseño lógico incluye un PLL que permite sintetizar los relojes de sistema y de muestreo a partir de la referencia de $10 \mathrm{MHz}$ provista por un TCXO. Para el reloj de sistema se utiliza una frecuencia de $80 \mathrm{MHz}$ mientras que el reloj de muestreo posee una frecuencia de $40 \mathrm{MHz}$. El primero temporiza el procesador LEON3 junto con el bus AMBA y todos los periféricos asociados. El reloj de muestreo, en cambio, temporiza los acumuladores de los canales de correlación del Módulo de Correlación y sus temporizadores asociados.

El módulo de correlación procesa las muestras provenientes de la etapa de RF. Su arquitectura interna se muestra en la Fig. 4. Cuenta con 24 canales para procesar señales de GPS en la banda de L1, 12 canales para GPS en banda L2 y un canal dedicado para la adquisición de señales L1. Este último permite detectar la presencia de un dado satélite en la señal recibida de forma rápida y obtener una primera estimación de los parámetros de su señal. Los temporizadores de adquisición y seguimiento controlan la cantidad de muestras procesadas por ciclo de correlación. Por otro lado, también se incluyen submódulos para controlar la señal de Pulso por Segundo (PPS) y un temporizador de tiempo real (RTC), cuya principal función es brindar soporte al software para etiquetar las mediciones de los canales de correlación.

Tanto los canales de L1 como los de L2 pueden procesar simultáneamente las señales de las dos antenas del receptor mientras que el canal de adquisición rápida puede procesar las señales de ambas antenas, pero no de forma simultánea. 


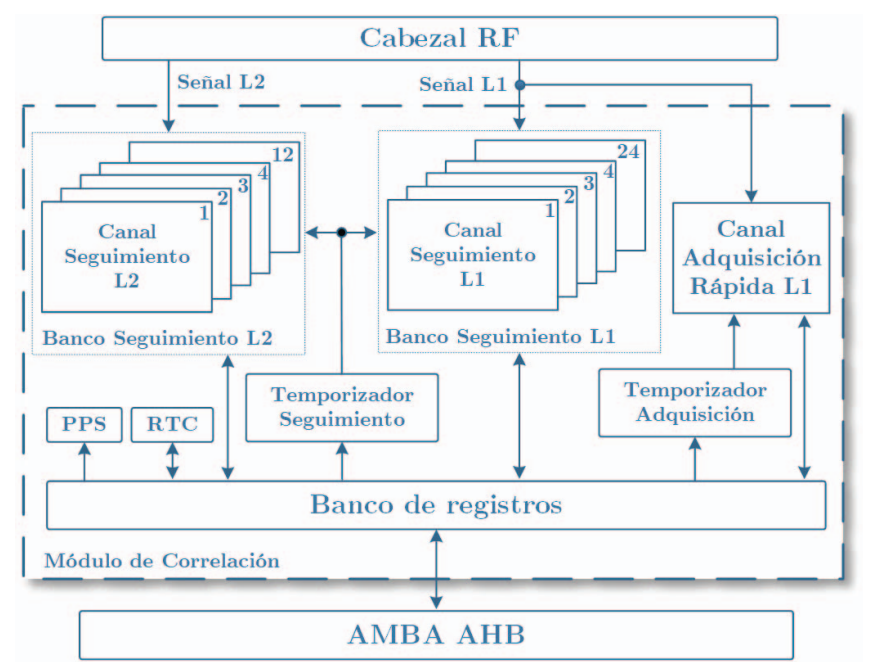

Fig. 4. Arquitectura interna del Módulo de Correlación.

El banco de registros contiene mapeados todos los parámetros de configuración de cada uno de los canales de correlación, así como los parámetros de los temporizadores y del submódulo PPS. Por otro lado, dicho banco también mapea los resultados de las correlaciones para que puedan ser accedidos y procesados por el software que se ejecuta en el LEON3.

\section{Diseño del Software}

El software del receptor está programado en lenguaje C sobre el sistema operativo de tiempo real RTEMS, lo que permite aprovechar las ventajas de un kernel multi-tarea de código abierto diseñado para sistemas embebidos y validado en múltiples plataformas e industrias, incluyendo la espacial. Basándose en el kernel mencionado, la estructura del software se dividió en un conjunto de módulos, cada uno compuesto por tareas gestionadas por el sistema operativo con distintas prioridades. La Fig. 5 muestra un diagrama en bloques del software, donde se visualizan dichos módulos y las interacciones entre los mismos y el hardware del receptor.

A continuación, se detallan brevemente las funciones de cada uno de los módulos por orden de mayor a menor prioridad.

El módulo de adquisición llevado a cabo por la tarea de mayor prioridad, es el encargado de la detección de las señales de los distintos satélites de GPS y la determinación de sus parámetros fundamentales (frecuencia y fase de portadora, fase de código, etc.).

El módulo de seguimiento se encarga del análisis de la evolución temporal de las señales adquiridas en el módulo anterior. Obtiene los datos del mensaje de navegación y genera mediciones de los tiempos de transmisión de estos mensajes.

Los dos módulos descritos anteriormente son coordinados por el módulo de gestión de señales. Este módulo solicita la adquisición de nuevas señales mientras haya canales de seguimiento disponibles, y comunica los parámetros determinados por las tareas de adquisición al módulo de seguimiento.

La demodulación de los datos del mensaje de navegación, generados por el módulo de seguimiento, es alimentada al

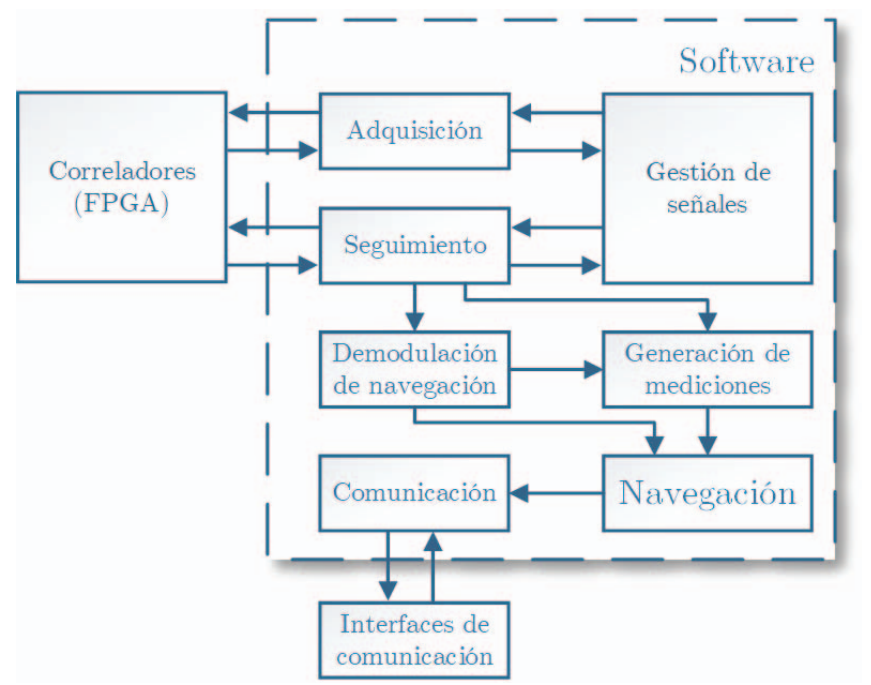

Fig. 5. Arquitectura de alto nivel del software.

módulo de demodulación de navegación, que se encarga de obtener las palabras transmitidas en el mensaje y desempaquetar la información orbital y de estado de los satélites contenida en las mismas. A partir de esta información y de los tiempos de transmisión (también estimados por el módulo de seguimiento), el receptor genera las mediciones de pseudorango y deltarango.

El módulo de navegación realiza la función principal del receptor: determinar la posición, velocidad y tiempo (también conocida como solución PVT) del usuario a partir de las mediciones de pseudorango y deltarango mencionadas anteriormente. La tarea encargada de esta función es llamada periódicamente por cada conjunto de mediciones generadas por el módulo anterior, y se encarga de descartar las mediciones de las cuales no conozca el estado de los satélites. Posteriormente, aplica las correcciones necesarias, como las del reloj de satélite y la de efectos ionosféricos y relativistas, a cada una de las restantes mediciones antes de emplearlas en el cálculo de la solución puntual de posición.

Finalmente, el módulo con menor prioridad es el encargado de transmitir toda la información generada por el receptor (solución de posición, mediciones crudas, información de estado y depuración, etc.) al usuario. Este módulo de comunicación se encuentra subdividido en tres niveles: una capa de protocolo, que organiza la información a enviar en distintos mensajes identificados por un campo particular; una capa de transporte que encapsula dichos mensajes en paquetes estructurados adecuados para el medio de comunicación por el que se va a utilizar; y una capa de dispositivo que se encarga de controlar a bajo nivel la interfaz de salida a utilizar para el envío de los paquetes. Esta división permite un nivel de abstracción que facilita la implementación de las distintas interfaces de salida del receptor (UART, CAN, I2C, etc.) proveyendo solo la última capa de cada una, mientras que permite el uso de estructuras de alto nivel, como colas de mensajes de entrada y de salida en las capas superiores. 


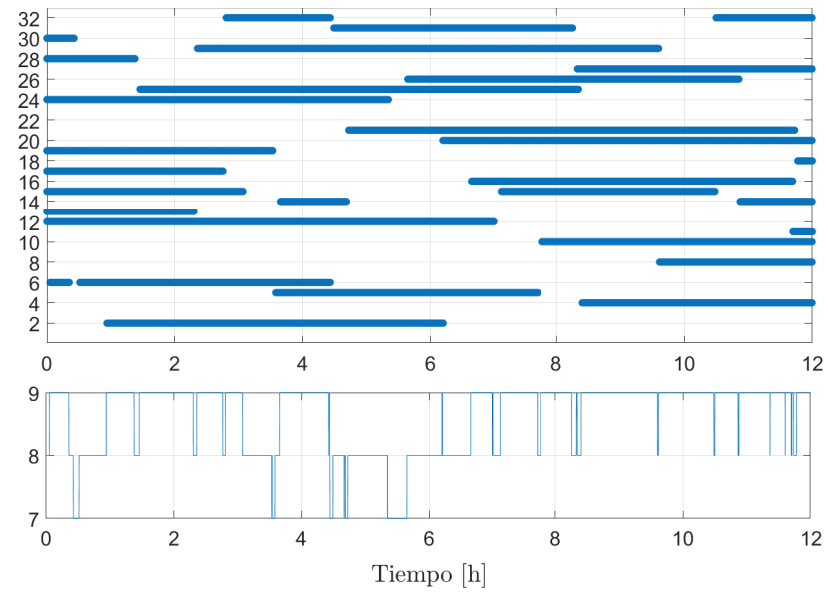

Fig. 6. Visibilidad de satélites GPS en función del tiempo (arriba) y cantidad de satélites en uso para el cálculo de la solución PVT (abajo) en el ensayo de posicionamiento absoluto.

\section{RESUltados}

A continuación, se exponen los resultados obtenidos a partir de tres ensayos diferentes, realizados con el fin de evaluar el desempeño de las principales funcionalidades del receptor que pueden ser de interés en distintas aplicaciones.

\section{A. Ensayo de Posicionamiento Absoluto}

Este ensayo consiste en la obtención del error en la solución de posición en coordenadas locales del receptor en una posición estática a partir de mediciones de pseudorango obtenidas de las señales GPS L1 por un lado, y GPS L1 y L2 en combinación libre de ionósfera por otro [18]. Los receptores GNSS suelen trabajar de manera natural con el marco de coordenadas ECEF (sistema de coordenadas centrado y fijado en el centro de la Tierra) por lo que para efectuar el cambio de coordenadas a un marco local East-North-Up (ENU) es necesario obtener la posición o una estimación de la posición del receptor [18]. Dicha posición fue determinada previamente mediante un receptor de alto desempeño utilizando el método de posicionamiento puntual preciso (PPP), el cual permite alcanzar precisiones del nivel de milímetros en receptores estáticos a costa de realizar un modelado exhaustivo de las mediciones de pseudorango y fase de portadora, de los fenómenos que las alteran y de disponer de productos orbitales de alta calidad [19].

El ensayo tuvo una duración de 12 horas y fue realizado en la terraza despejada del edificio del Departamento de Electrotecnia de la Facultad de Ingeniería de la Universidad Nacional de La Plata (UNLP), por lo que la visibilidad de satélites era óptima como puede observarse en la Fig. 6. En las Figs. 7 y 8 se muestra el error de posición para GPS L1 y GPS L1+L2 respectivamente. Para el caso de GPS L1 puede notarse que las variaciones del error en el plano horizontal E-N están acotadas en 2 metros, mientras que la vertical, que es la coordenada más afectada por la disposición geométrica de la constelación, está acotada en 6 metros. Estos resultados advierten sobre la buena calidad de la solución obtenida, ya que el nivel de ruido de la misma es equiparable al de un receptor del tipo geodésico, que son los de la mejor calidad
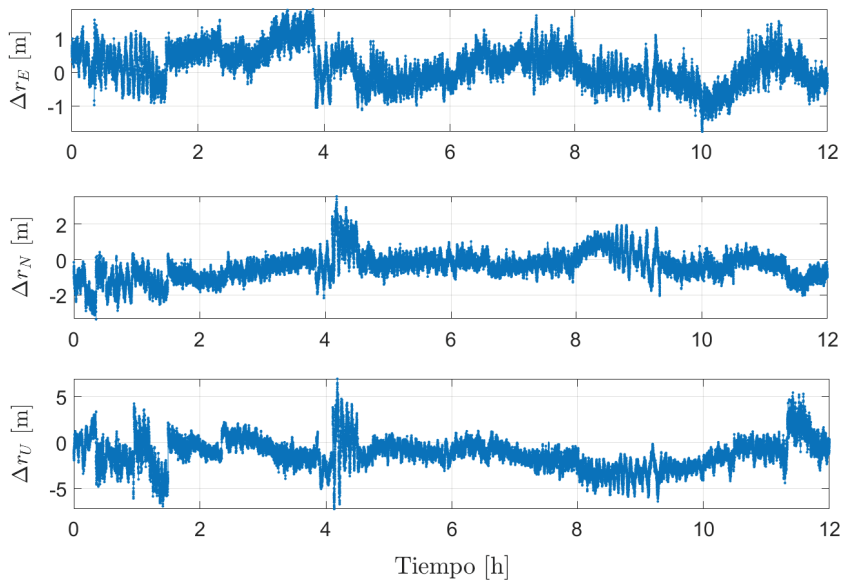

Fig. 7. Error de posición en la solución interna de navegación GPS L1.
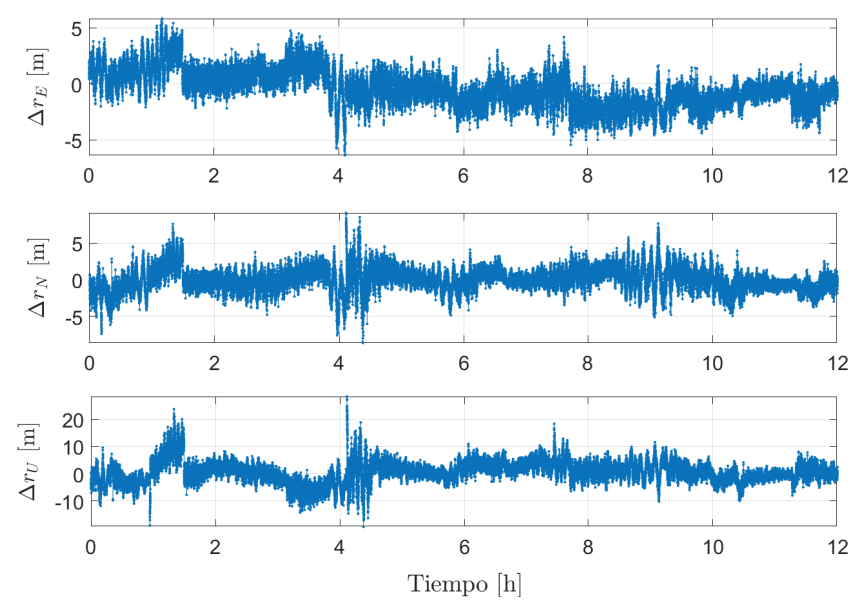

Fig. 8. Error de posición en la solución interna de navegación GPS L1 y L2.

disponibles actualmente en el mercado [18]. Para el caso doble frecuencia y combinación libre de ionósfera de los satélites que transmiten ambas señales (Fig. 8) se observa que el nivel de ruido es mayor debido a que la combinación enaltece la varianza de las mediciones de pseudorango [8].

En la Tabla I se detallan la media y la desviación estándar del error para ambos casos. Nótese que la disminución en la media para el caso $\mathrm{L} 1+\mathrm{L} 2$, en particular en la componente vertical, es coherente con la eliminación práctica del retardo ionosférico al utilizar las mediciones de dos frecuencias combinadas. Para el caso de L1, el retardo ionosférico se descuenta con un modelo que no es tan efectivo como la combinación del caso doble frecuencia. Cabe destacar que los resultados presentados en las Figs. 7 y 8 corresponden a la solución puntual obtenida para cada instante y no poseen filtrado alguno, por lo que es de esperar una notable mejora en caso de filtrar adecuadamente la solución obtenida, en particular para el caso $\mathrm{L} 1+\mathrm{L} 2$.

\section{B. Ensayo de Posicionamiento Diferencial}

Teniendo en consideración la posibilidad del uso de este tipo de receptores para misiones espaciales de más de un nanosatélite, o incluso aplicaciones terrestres de alta precisión, es de particular interés que el receptor cumpla las condiciones 
TABLA I

Resultados de Posicionamiento Absoluto

\begin{tabular}{cccc}
\hline \hline $\begin{array}{c}\text { Modo de } \\
\text { procesamiento }\end{array}$ & Coordenadas & Media [m] & Desv. Est. [m] \\
\hline \multirow{2}{*}{ L1 } & $\mathrm{E}$ & 0.161 & 0.5 \\
& $\mathrm{~N}$ & -0.376 & 0.7 \\
& $\mathrm{U}$ & -1.238 & 1.6 \\
$\mathrm{~L} 1+\mathrm{L} 2$ & $\mathrm{E}$ & -0.445 & 1.6 \\
& $\mathrm{~N}$ & 0.001 & 1.6 \\
& $\mathrm{U}$ & -0.521 & 3.9 \\
\hline \hline
\end{tabular}

necesarias para la determinación precisa de línea base mediante el uso de mediciones de fase de portadora [20].

Con este fin se llevó a cabo un ensayo de posicionamiento diferencial en tierra estático, utilizando como referencia un receptor de características similares al expuesto anteriormente [21], a excepción de su etapa de RF, apropiada para posicionamiento de alta precisión debido a su baja figura de ruido. El objetivo de este ensayo fue asegurar la capacidad del receptor de proporcionar mediciones precisas de fase de portadora aptas para su utilización en algoritmos de posicionamiento mediante resolución de las ambigüedades enteras de longitud de onda completa.

La línea base estaba conformada por dos antenas distantes unos 27 metros entre sí, en condiciones de buena visibilidad, aunque no inmunes a efectos de multicamino. Se tomaron mediciones de pseudorangos y fases de portadora de GPS en bandas L1 y L2 con intervalo de muestreo de 1 segundo durante 2 horas, las cuales fueron almacenadas para su posterior procesamiento offline.

El diseño de ambos receptores con sus esquemas de correlaciones de duración variable permite contar con mediciones alineadas al tiempo GPS aptas directamente para su procesamiento diferencial. Sin embargo, para esto el receptor debe primero adquirir al menos 4 satélites con el fin de resolver su posición y sesgo de reloj. Por ello, el primer paso de procesamiento consiste en la eliminación de las primeras épocas aún no alineadas al tiempo GPS.

El esquema de estimación empleado fue un filtro de Kalman, utilizando como mediciones propiamente dichas las dobles diferencias de pseudorangos y fases de portadora en cada frecuencia disponible por satélite. Esto permite la cancelación de errores comunes tanto a los satélites como así también a cada receptor. Los estados a estimar fueron las tres coordenadas del vector línea base y las ambigüedades flotantes de longitud de onda, es decir estimadas como números reales. Una vez realizada la actualización observacional del filtro en cada época, se utilizó esta estimación flotante de las ambigüedades y su matriz de covarianza para realizar la resolución de las mismas mediante el llamado método LAMBDA [22], y resolver nuevamente el sistema lineal mediante mínimos cuadrados pesados con las ambigüedades resueltas.

Dado que el receptor es capaz de seguir el código C/A en banda L1 y el código L2C en banda L2, el cual no es transmitido actualmente por todos los satélites, se llevó a cabo una implementación que permite el uso de las mediciones
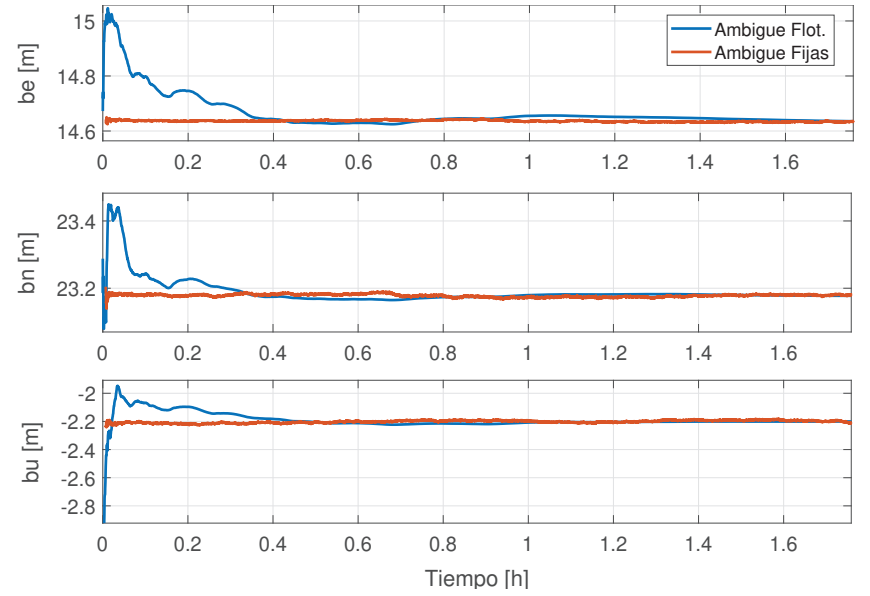

Fig. 9. Línea base estimada por el filtro de ambigüedades flotantes (azul) y por solución puntual luego de resolver ambigüedades (naranja). Coordenadas locales East-North-Up (ENU).

TABLA II

Resultados de Posicionamiento Diferencial

\begin{tabular}{cccc}
\hline \hline $\begin{array}{c}\text { Modo de } \\
\text { procesamiento }\end{array}$ & Coordenadas & Media [m] & Desv. Est. [m] \\
\hline \multirow{3}{*}{ Amb. Flot } & $\mathrm{E}$ & 14.645 & 14.2 \\
& $\mathrm{~N}$ & 23.117 & 7.4 \\
& $\mathrm{U}$ & -2.202 & 16.5 \\
Amb. Res. & $\mathrm{E}$ & 14.636 & 3.0 \\
& $\mathrm{~N}$ & 23.178 & 4.3 \\
& $\mathrm{U}$ & -2.202 & 9.1 \\
\hline \hline
\end{tabular}

disponibles por cada satélite. Esto implica que no todos los satélites cuentan con mediciones en las dos frecuencias.

La Fig. 9 muestra el resultado de la estimación de la línea base. Una vez pasado el transitorio inicial, de aproximadamente 10 minutos, el filtro de ambigüedades flotantes converge a una solución y se estabiliza. De mayor importancia aún, se observó que luego de 25 segundos el sistema es capaz de resolver correctamente las ambigüedades, sin presentarse ningún salto de ciclo ni resolución errónea de las mismas, posibilitando el uso de las fases de portadora como pseudorangos de alta precisión.

La Tabla II resume los resultados obtenidos tanto a partir de la solución del filtro con ambigüedades flotantes, como la obtenida época a época posterior a la resolución de las ambigüedades. Para el cálculo de los parámetros de la solución flotante se excluyeron los primeros 15 minutos de forma de eliminar el transitorio inicial. Puede observarse que ambas soluciones coinciden en media al nivel de los milímetros. Por otra parte, una vez resueltas las ambigüedades se logra una precisión del orden del centímetro (3D), lo cual permite validar el uso del receptor para este tipo de aplicaciones y algoritmos de alta precisión. Cabe destacar que el esquema de posprocesamiento utilizado en este ensayo tiene como objetivo únicamente demostrar el potencial del receptor para aplicaciones de alta precisión, ya que este método es inviable para tiempo real. En ese aspecto actualmente se está trabajando en base a técnicas y resultados presentados en [23]. 


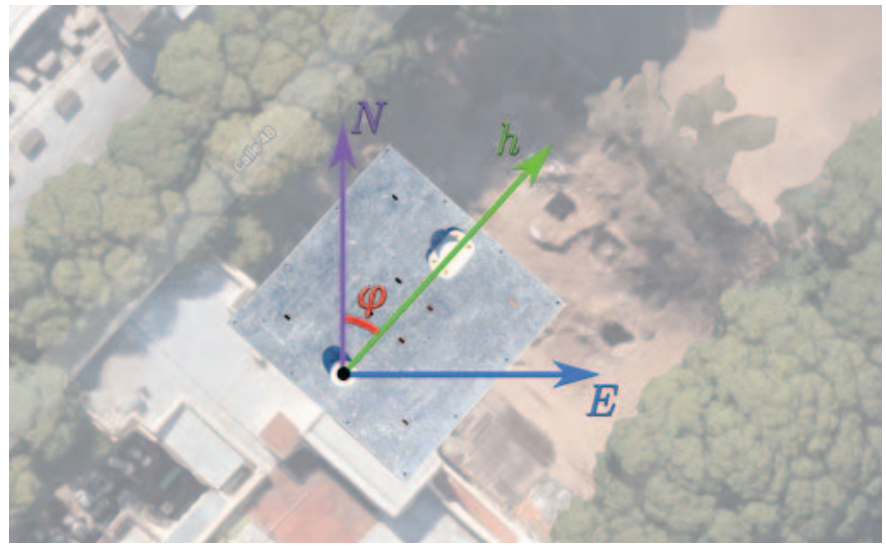

Fig. 10. Orientación del arreglo hacia el noreste. El arreglo no se encuentra a escala para mejorar la visibilidad.

\section{Ensayo de Orientación Parcial Estática}

Con el fin de conferir una nueva funcionalidad al receptor y aprovechando la capacidad de procesar señales de dos antenas independientes es posible calcular la orientación de un arreglo de dos antenas utilizando mediciones GNSS. En particular se utilizan mediciones de fase de GPS L1 y L2. Debido a que la diferencia de fase con que arriba la señal a cada antena guarda relación con el resultado de las correlaciones que calcula periódicamente el receptor, es posible utilizar dichas correlaciones para inferir la orientación parcial del arreglo en el plano horizontal en coordenadas ENU. El método utilizado para obtener las mediciones del receptor, como así también el esquema de procesamiento elegido, se detallan en [24].

En particular el ensayo realizado consistió en la toma de mediciones de fase de las señales GPS en las bandas L1 y L2 por cada antena durante unas 18 horas. El arreglo de antenas estaba formado por una chapa galvanizada de $60 \times 60$ $\mathrm{cm}$. En el centro de la chapa se colocaron dos antenas de GPS L1/L2 con una separación aproximada de $40 \mathrm{~cm}$. Durante el ensayo el arreglo se mantuvo en posición estática y se orientó aproximadamente hacia el noreste, como se muestra en la Fig. 10.

Se realizó el procesamiento utilizando un filtro de Kalman, de manera similar al utilizado en el ensayo de posicionamiento diferencial, pero utilizando como mediciones las diferencias de fase inferidas a partir del resultado de las correlaciones [24]. A partir de la estimación de la línea de base, que es la línea que une ambas antenas, fue posible estimar la orientación del arreglo en cabeceo (o yaw) y guiñada (o pitch). Debido al posicionamiento del arreglo se esperaba hallar un ángulo de cabeceo cercano a $45^{\circ}$ y un ángulo de guiñada aproximadamente nulo. En la Fig. 11 se muestran las coordenadas de línea de base estimadas en marco local junto al módulo, el cual puede notarse que, luego de converger el filtro, es de aproximadamente $40 \mathrm{~cm}$. En la Fig. 12 se observa el cálculo del ángulo de cabeceo y guiñada a partir de las coordenadas de línea de base. Puede apreciarse que los apartamientos de los valores esperados se mantienen dentro de límites razonables (menos de $10^{\circ}$ ) para el cabeceo, que es el más afectado en la estimación debido a su dependencia con la
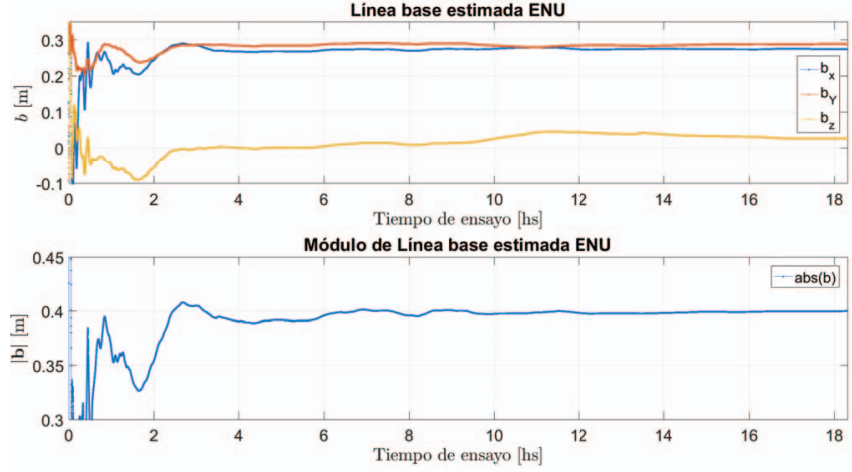

Fig. 11. Coordenadas de la línea de base en marco ENU. Debajo se grafica el módulo de la línea, el cual es aproximadamente $40 \mathrm{~cm}$, resultado esperado que concuerda con la separación física entre las antenas.
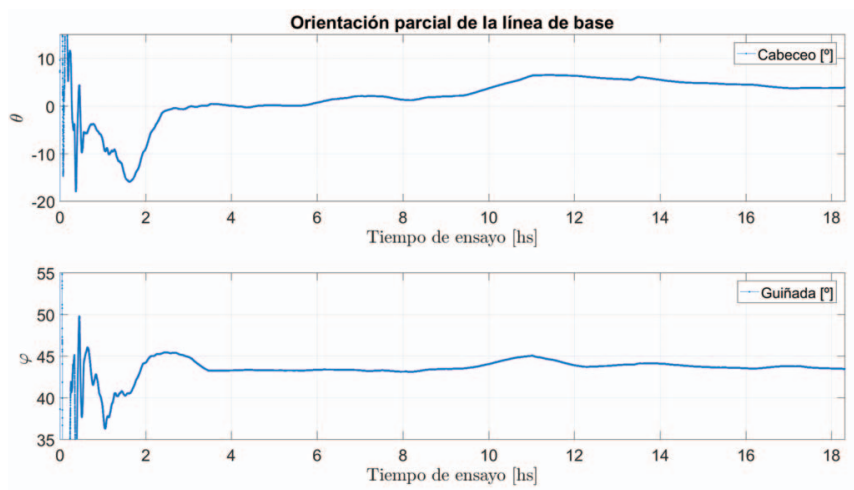

Fig. 12. Ángulos de orientación parcial a partir de la estimación de la línea de base. El ángulo de cabeceo (arriba) o pitch es el medido entre el plano horizontal y el arreglo. El ángulo de guiñada (abajo) o yaw es considerado como se muestra en la Fig. 10.

componente vertical. Al igual que en el ensayo anterior, estos resultados obtenidos de manera offline, permiten mostrar el potencial uso del cálculo de orientación como característica adicional del receptor GPS. Actualmente se está trabajando en la implementación en tiempo real de esta funcionalidad utilizando la técnica presentada en [25].

\section{CONCLUSiOnes}

Se presentó el diseño y la implementación de un receptor GPS doble banda y doble antena. Se describieron los aspectos más destacados de la construcción física de un prototipo como así también del diseño lógico digital y del software que se ejecuta en el receptor. Con el fin de evaluar el desempeño se realizaron tres ensayos funcionales de particular interés para este tipo de receptores: el primero de posicionamiento puntual absoluto, el segundo con posicionamiento estático diferencial y, finalmente para determinar la orientación parcial de un arreglo de dos antenas estático. Los resultados obtenidos en los dos primeros ensayos permitieron constatar la buena calidad de las mediciones que otorga el receptor, comparables con las de un receptor geodésico. A su vez, el ensayo de orientación parcial permitió validar una característica funcional extra sumamente útil, por ejemplo, en la asistencia al sistema de navegación de un vehículo para obtener una estimación de la orientación 
absoluta sin demasiada complejidad o capacidad de cómputo necesaria desde el punto de vista del procesamiento que realiza el receptor.

\section{REFERENCIAS}

[1] A. Toorian, K. Diaz and S. Lee, "The CubeSat Approach to Space Access," in 2008 IEEE Aerospace Conference, Big Sky, MT, USA, 2008, pp. 1-14.

[2] D. Selva and D. Krejcib, "A survey and Assessment of the Capabilities of Cubesats for Earth Observation," Acta Astronautica, vol. 17, pp. 50-68, May-June 2012

[3] S.R. Tsitas and J. Kingston, "6U CubeSat Commercial Applications," The Aeronautical Journal, vol. 116, no. 1176, pp. 189-198, Feb. 2012.

[4] K. Woellert, P.Ehrenfreund, A. Ricco, and H. Hertzfeld, "Cubesats: Costeffective science and Technology platforms for emerging and developing Nations," Advances in Space Research, vol. 47, no. 4, pp. 663-684, Feb. 2011.

[5] J. Puig-Suari, C. Turner, and W. Ahlgren, "Development of the Standard CubeSat Deployer and a CubeSat Class PicoSatellite," in 2001 IEEE Aerospace Conference Proceedings, Big Sky, MT, USA, 2001, pp. 1/347$1 / 353$.

[6] M. Swartwout, "The First One Hundred CubeSats: A Statistical Look," Journal of Small Satellites, vol. 2, no. 2, pp. 213-233, Dic. 2013.

[7] E. Peral et al., "Radar Technologies for Earth Remote Sensing From CubeSat Platforms," Proceedings of the IEEE, vol. 106, no. 3, pp. 404418, March 2018

[8] E. D. Kaplan and C. Hegarty, Understanding GPS/GNSS: Principles and Applications, 3rd ed. Norwood, MA, USA: Artech House, 2017.

[9] NewSpace Systems, "NGPS-01-422 GPS Receiver," South Africa

[10] Surrey Satellite Technology Ltd, "Space GNSS Receiver SGR-Ligo," UK.

[11] Pumpkin Inc., “CubeSat Kit GPSRM 1 GPS Receiver Module," rev. D, USA, 2014.

[12] Hyperion Technologies, "GNSS200 Series Global Navigation Receiver," Netherlands, 2018.

[13] Y. T. James Bao, Fundamentals of Global Positioning System Receivers: A software Approach, Hoboken, NJ, USA: John Wiley \& Sons, 2005.

[14] Pumpkin Inc., “CubeSat Kit PCB Specificaction,” USA, 2003.

[15] H. Ott, Electromagnetic Compatibility Engineering, Hoboken, NJ, USA John Wiley \& Sons, 2009.

[16] Cobham plc, "GRLIB IP Library User's Manual," v. 2018.1, UK, 2018.

[17] J. G. Díaz, Germán Scillone, J. G. García, "Sistema de Adquisición en FPGA para receptores SDR", 26 Congreso Argentino de Control Automático (AADECA'18), Buenos Aires, Argentina, pp. 440-445.

[18] P. Teunissen and O. Montenbruck, Springer Handbook of Global Navigation Satellite Systems, Springer, 2017.

[19] J. F. Zumberge, M. B. Heflin, D. C. Jefferson, M. M. Watkins and F. H. Webb, "Precise Point Positioning for the Efficient and Robust Analysis of GPS Data from Large Networks," Journal of Geophysical Research, vol. 102, pp. 5005-5017, March 1997.

[20] M. L. Psiaki and S. Mohiuddin, "Modeling, Analysis and simulation of GPS Carrier-Phase for Spacecraft Relative Navigation," Journal of Guidance, Control, and Dynamics, vol. 30, no. 6, pp. 1628-1639, Dic. 2007.

[21] J. G. Díaz, R. López La Valle, S. Rodríguez, G. Scillone, L. Martire, E. M. López, G. L. Puga, J. Cogo, J. A. Smidt, J. G. García, P. A Roncagliolo, "An Experimental High Precision GNSS Receiver for Small Satellites Navigation," in 1st IAA Latin American Symposium on Small Satellites, Buenos Aires, Argentina, 2017.

[22] P. De Jonge, C. Tiberius, "The LAMBDA Method for Integer Ambiguity Estimation: Implementation Aspects," Delft Geodetics Computing Centre, Delft, Netherlands, Aug. 1996.

[23] J. G. García, P. A. Roncagliolo, C. H. Muravchik, "A Bayesian Technique for Real and Integer Parameters Estimation in Linear Models and Its Application to GNSS High Precision Positioning," IEEE Transactions on Signal Processing, vol. 64, no. 4, pp. 923-933, Feb. 2016.

[24] S. Rodríguez, E. M. López, J. G. García, P. A. Roncagliolo, "Estimación de Orientación Parcial Estática de un Arreglo de Antenas con Mediciones GNSS," in XVIII Reunión de Trabajo en Procesamiento de la Información y Control (RPIC 2019), Buenos Aires, Argentina, 2019.

[25] G. García, P. Axelrad, P. A. Roncagliolo, C. H. Muravchik, "Fast and Reliable GNSS Attitude Estimation Using a Constrained Bayesian Ambiguity Resolution Technique (C-BART)," in 28th International Technical Meeting of the Satellite Division of The Institute of Navigation (ION GNSS+ 2015), Tampa, FL, September 2015, pp. 2809-2820.

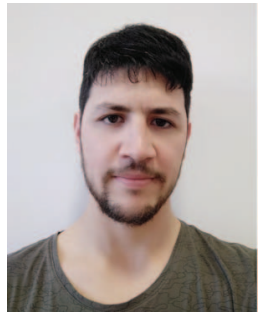

Santiago Rodríguez received the Electronics Engineering degree from the National University of La Plata (UNLP), Argentina, in 2013. He is currently an Assistant Professor in the UNLP, involved in research and development of Global Navigation Satellite Systems (GNSS) receivers and processing techniques for aerospace and high-precision applications. His interests are in hardware and PCB design for digital processing and Embedded Systems with applications to GNSS.

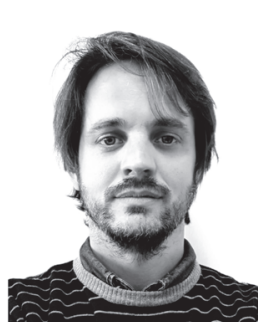

Javier G. García received the Electronics Engineering degree from the National University of La Plata (UNLP), Argentina, in 2003. He is currently a Professor in the UNLP, involved in research and development of Global Navigation Satellite Systems (GNSS) receivers and processing techniques for aerospace and high-precision applications. His interests are in Statistical Signal Processing, Digital Communications, and Embedded Systems with applications to GNSS.

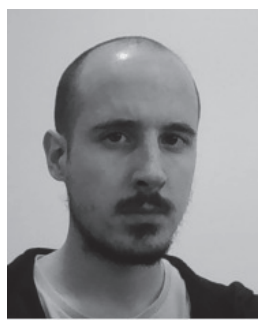

Germán Scillone was born in Azul, Buenos Aires, Argentina in 1990. He received the Electronics Engineering degree from the National University of La Plata (UNLP) in 2015. Since then, he has been developing and maintaining software for various GNSS related projects at SENyT, with a focus on real-time embedded systems for critical applications.

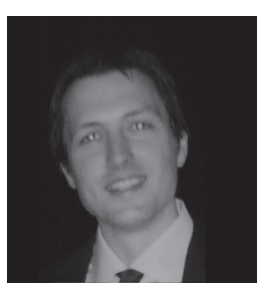

Juan G. Díaz was born in La Plata in 1985 . He received the Electronic Engineering degree in 2011 from the National University of La Plata (UNLP) and currently he is finishing a Magister in Engineering at the UNLP. He is Teacher Assistant in the UNLP and he works in the SENyT in topics related to develop of Global Navigation Satellite Systems receivers in the digital design and embedded systems areas.

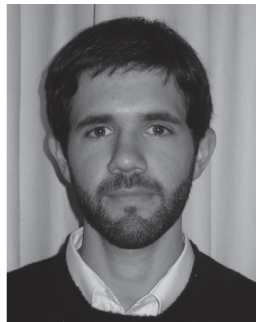

Ernesto M. López received the Electronics Engineering degree from the National University of La Plata (UNLP), Argentina in 2015. He is currently pursuing the Ph.D. degree in Electronics Engineering at UNLP. His research interest includes statistical signal processing applied to the development of high precision GNSS-based positioning techniques, and precise orbit and baseline determination for satellite navigation.

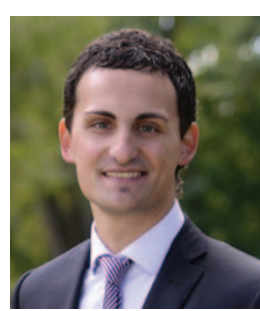

Ramón López La Valle received the Electronics Engineering and the MSc in Engineering degrees from the National University of La Plata (UNLP), Argentina, in 2008 and 2014. He is an Assistant Professor in the UNLP involved in research and development of Global Navigation Satellite Systems (GNSS) receivers for aerospace applications. His research interests are in electromagnetic compatibility, antennas, and RF and microwave circuits with applications to GNSS and wireless communications. 